\title{
Biomolecules Behavior on a Surface of Boron Doped/un-doped Graphene Nanosheets
}

\author{
Abdelfattah Amari ${ }^{1,2}$, Basem Alalwan ${ }^{1}$, Saifeldin M. Siddeeg ${ }^{3,4}$, Mohamed A. Tahoon $^{3, *}$, \\ Norah Salem Alsaiari, and Faouzi Ben Rebah ${ }^{3,6}$ \\ ${ }^{1}$ Department of Chemical Engineering, College of Engineering, King Khalid University, PO Box \\ 9004, Abha 61413, Saudi Arabia \\ ${ }^{2}$ Research Laboratory: Energy and Environment, National School of Engineers, Gabes University, \\ Gabes 6072, Tunisia \\ ${ }^{3}$ Department of Chemistry, College of Science, King Khalid University, P.O. Box 9004, Abha 61413, \\ Kingdom of Saudi Arabia \\ ${ }^{4}$ Chemistry and Nuclear Physics Institute, Atomic Energy Commission, P.O. Box 3001, Khartoum \\ 11111, Sudan \\ ${ }^{5}$ Chemistry Department, College of Science, Princess Nourah Bint Abdulrahman University, Riyadh, \\ Saudi Arabia \\ ${ }^{6}$ Higher Institute of Biotechnology of Sfax (ISBS), Sfax University, P.O. Box 263, Sfax 3000, Tunisia \\ *E-mail: tahooon_87@yahoo.com
}

doi: $10.20964 / 2020.11 .37$

Received: 6 June 2020 / Accepted: 23 July 2020 / Published: 30 September 2020

Recently, carbon-doped nanomaterials attract attention due to their unconventional properties as doping completely changes their properties. Herein, we try to compare the electrochemical response of the prepared boron-doped graphene nanosheets and un-doped graphene nanosheets modified glassy carbon electrodes toward some interesting biomolecules like ascorbic acid, uric acid, and dopamine. There are significant differences of prepared doped and un-doped graphene nanosheets toward the mentioned biomolecules. The resistance of surfaces toward fouling was studied on uric acid oxidation product in the long term. In the long term stability of the prepared materials, there are no significant differences found. As electrochemical materials, the differences between prepared materials will affect their applications.

Keywords: Biomolecules; Boron Doped graphene nanosheets; Biosensors 
(C) 2020 The Authors. Published by ESG (www.electrochemsci.org). This article is an open access article distributed under the terms and conditions of the Creative Commons Attribution license (http://creativecommons.org/licenses/by/4.0/). 